\title{
A Brief Study of Physical Properties of Rf Magnetron Sputtered Azo Thin Films
}

\author{
Richa Sharma $^{1 *} \bullet{\text { Fouran } \operatorname{Singh}^{2} \bullet \text { J M S Rana }}^{1}$ \\ ${ }^{1}$ Department of Physics, H.N.B Garhwal University, SRT Campus Badshahi Thaul, Tehri Garhwal 249199 \\ ${ }^{2}$ Materials Science Group, Inter University Accelerator Centre, Aruna Asaf Ali Marg New Delhi 110067 \\ *Corresponding Author Email: sharmariccha05@gmail.com
}

Received: 4.9.2021; Revised: 19.10.2021; Accepted: 28.10.2021

(C) Society for Himalayan Action Research and Development

\begin{abstract}
We reported the physical properties of undoped and Aluminium doped ZnO (AZO) thin films that were synthesized by the RF magnetron sputtering method on a quartz substrate. The effect of dopant concentration on structure, morphology, optical and electrical properties of the thin films have been studied systematically by XRD, Raman spectroscopy, AFM, FE-SEM, UV-VIS spectroscopy and I-V measurement (two probe method) respectively. The crystallite growth of the thin films is along c-axis (002) orientations with hexagonal wurtzite structure. The crystallinity is enhanced in the $1 \% \mathrm{AZO}$ thin films as compared to undoped $\mathrm{ZnO}$. The transition of stress value after introducing the dopant is discussed. These changes are further correlated with the observed morphological changes. The alteration in optical transmission and optical band gap is also discussed extensively. For the AZO (1\% and 2\%) thin films the n-type conductivity and ohmic nature measured by using Keithley two probe set up. The responsible mechanism for improved conductivity is discussed. In the FTIR spectrum the peaks originated by the tetrahedral coordination of $\mathrm{ZnO}$ are observed which further confirms the wurtzite structure of the deposited thin film sample that are recorded by the XRD pattern.
\end{abstract}

Keywords: RF magnetron sputtering, FE-SEM, Raman Spectroscopy, AZO and optical bandgap.

\section{Introduction}

The economical, inert and accessible metal oxides have been extensively studied for commercial applications. In the fashion of miniaturization, the study of dimensionality has also been broadly explored by the scientific community for their possible applications such as electrochromic windows(Granqvist, 2000) and optoelectronic devices(Malek et al., 2013; Peiró et al., 2006). The requirement of these high-performance devices is contented by the metal oxide Zinc oxide $(\mathrm{ZnO})$ that have good structural, electrical, optical and electrochemical properties. The resistive stoichiometry of $\mathrm{ZnO}$ can be converted to conducting nature by introducing oxygen vacancies that behave as donors with a proper dopant. The group III elements such as aluminium (Al) are chosen as dopant because $\mathrm{Al}$ has much smaller ionic radii $(0.54 \AA)$ as compared to $\mathrm{Zn}(0.74 \AA)$ that can favourably occupy the interstitial(Lee and Park, 2004).

The thin film growth technique and conditions strongly affect the crystal structure. In the past time, researchers are using topdown approaches and methodologies to achieve high-quality $\mathrm{ZnO}$ thin films. However, the commercial application oriented devices require a low cost but necessarily with high- 
performance and reproducibility. While choosing the appropriate technique as per the requirement a thorough analysis of the external factors should also be considered. The literature of $\mathrm{ZnO}$ thin films includes many different thin film deposition techniques. The basic material properties of the $\mathrm{ZnO}$ material can be augmented and some functionality could also be added by choosing different deposition techniques in addition to other material tailoring methods (doping, annealing, different substrate and ion irradiation etc.). RF magnetron sputtering is one of such methods to produce good quality thin films that provide controlled composition, homogeneity, great adhesion and control of thin-film thickness. In the fabrication of devices, the present stress/ strain in the AZO thin film is expected to be a critical factor that influences its performance. Though there are already plenty of reports on AZO thin films, relatively a few focus on the effect of the stress/ strain and their correlation with the other material properties. The authors decisively believe that such a study is beneficial to deepen the understanding for the development of high-quality thin films for improved $\mathrm{ZnO}$ based devices.

\section{Experimental details}

The undoped $\mathrm{ZnO}$ and $\mathrm{Al}$ doped thin films with aluminium content $1 \%$ AZO and $2 \% \mathrm{AZO}$ were deposited using RF magnetron sputtering technique at room temperature on quartz and the silicon substrate. The targets were prepared by employing the solid-state reaction method. A rough vacuum is created in the sputtering chamber by using a rotary pump through a roughing valve. Before introducing the gas in the chamber the pressure of the chamber is brought down to $5.5 \times 10^{-5}$ mbar. The constant flow of Argon gas in standard centimetre per minute $(\mathrm{sccm})$ is ensured by using MKS mass flow meters. The RF power was maintained at $100 \mathrm{~W}$ with a base pressure of $5 \times 10^{-2}$ mbar during the deposition. A high vacuum turbopump is used to achieve and maintain this low pressure. The separation between the target and substrate was $11 \mathrm{~cm}$. The post-annealing of undoped $\mathrm{ZnO}$ and $\mathrm{AZO}$ thin films is done in the oxygen environment for $1 \mathrm{hr}$ at $600^{\circ} \mathrm{C}$ using a microprocessorcontrolled furnace (GERO HRTH 40100/17, Nabertherm). This annealing is carried out to get thin films with good crystallinity and improved stoichiometry.

The crystallinity and structure of undoped $\mathrm{ZnO}$ and $\mathrm{AZO}$ thin films have been characterized at room temperature by using a glancing angle X-ray diffractometer (GAXRD), using Bruker D8 diffractometer with $\mathrm{Cu}-\mathrm{Ka} \mathrm{X}$-ray (1- $1.54 \AA$ ) for $2 \theta$ range from $20^{\circ}$ to $60^{\circ}$ with a scan rate of $0.2 / \mathrm{m}$. The structural studies were further done by the micro-Raman spectroscopy using a Renishaw In-Via Raman microscope. The 50mWArb ion laser in the $514.5 \mathrm{~nm}$ line is used to record the spectra. The microstructure and morphological investigations were done by a Scanning Electron Microscopy (SEM). Optical transmittance and absorbance spectra from 200 to $800 \mathrm{~nm}$ wavelength range were recorded using Hitachi UV 3300 double-beam spectrometer. The electrical transport 
properties are studied by the two probe method. The FTIR spectroscopy is employed to characterise the functional groups, vibrational properties, defect and impurities of the synthesized materials. The FTIR setup from Bruker (VORTEX70) is used for the measurement in the range of $200-4000 \mathrm{~cm}^{-1}$.

\section{Results and discussions}

\subsection{Structural properties}

In the present study, the thin films prepared by sputtering technique with different targets and are highly textured. The GAXRD patterns of the undoped, $1 \% \mathrm{AZO}$ and $2 \% \mathrm{AZO}$ thin film samples are represented in figure1. The X'pert high score software is used to appraise the recorded pattern. The peaks are indexed to the $\mathrm{ZnO}$ wurtzite structure (JCPDS card No. 36-1451). The pattern confirms the polycrystalline nature of the $\mathrm{ZnO}$ thin films with major diffraction from (002) peak with a c-axis orientation perpendicular to the substrate. The absence of the metallic peaks corresponding to the $\mathrm{Zn}$ or $\mathrm{Al}$ confirms the dopant is well substituted on the host site without causing much stress in the deposited thin films.

The preferred c-axis orientation of the thin film can be explained by the model "survival of the fastest" proposed by Drift (Van der Drift, 1967). The model explains that in the initial stage of deposition the nucleation of the various orientation is expected to be formed and every nucleus strives to grow but the nuclei with fastest growth rate can persist, i.e., c axis orientation is realized in $\mathrm{ZnO}$. The pattern reveals the presence of weak (102) peak, which evolve in $2 \% \mathrm{AZO}$ thin films as compared with undoped $\mathrm{ZnO}$ thin films. In $2 \%$ AZO thin film, sample have a mixed orientation with additional (100) and (101) peak of $\mathrm{ZnO}$ wurtzite structure.

Table 1: Full width at half maxima (FWHM), Crystallite Size (D), Dislocation density (ס), Strain $(\varepsilon)$, Stress $(\sigma)$ and Lattice parameter (c) for 1\%AZO and $2 \% A Z O$ thin film sample.

\begin{tabular}{|c|c|c|c|c|c|c|}
\hline Sample & $\begin{array}{c}\text { FWHM } \\
(002)\end{array}$ & $\mathbf{D}(\mathbf{n m})$ & $\begin{array}{c}\delta \\
\left(10^{14} \text { lines } \mathrm{m}^{-}\right. \\
2)\end{array}$ & $\begin{array}{l}\text { Strain } \\
\varepsilon\left(10^{-4}\right)\end{array}$ & $\begin{array}{c}\text { Stress } \\
\sigma(\mathbf{G P a})\end{array}$ & $\begin{array}{c}\text { Lattice } \\
\text { parameters } \\
\text { c( }(\AA)\end{array}$ \\
\hline $\begin{array}{c}\text { Undoped } \\
\mathrm{ZnO}\end{array}$ & 0.336 & 24.7 & 16.3 & 58.8 & -0.137 & 5.209 \\
\hline $1 \% \mathrm{AZO}$ & 0.312 & 26.6 & 14.1 & 57.7 & -0.135 & 5.209 \\
\hline $2 \% \mathrm{AZO}$ & 0.336 & 24.7 & 16.3 & -136.2 & 0.317 & 5.198 \\
\hline
\end{tabular}

The (002) reflection peak position for the deposited thin films is close to the standard value $\left(34.47^{0}\right)$ for $\mathrm{ZnO}$ powder. The effect of Al doping concentration can be seen with a 
shift in the (002) peak position from $34.44^{0}$ to $34.50^{\circ}$ for the $1 \% \mathrm{AZO}$ and $2 \% \mathrm{AZO}$ thin films. Part et al.,(Kim et al., 1997) also witnessed the shift in (002) peak towards the higher value similar to our observations. This shift can be attributed to the difference in the ionic radii of the host $\mathrm{Zn}^{2+}(0.74 \AA)$ and dopant $\mathrm{Al}^{3+}(0.54 \AA)$, which causes the shortening of the c-axis. The value of the lattice parameter $\mathrm{c}$ has been calculated for undoped $\mathrm{ZnO}, 1 \% \mathrm{AZO}$ and $2 \%$ AZO thin films and tabulated in Table1. The lattice parameter is calculated by using the following formula(Kaur et al., 2015)

$$
c=\frac{\lambda}{\sin \theta_{(002)}}
$$

These values are used for the clear estimation of the strain and stress in the thin film induced by the shortening of the $\mathrm{c}$ axis. The induced microstrain $(\varepsilon)$ of the thin films have been calculated using the following formula(Yilmaz et al., 2016):

$\varepsilon=\frac{\beta}{4 \tan \theta}$ 2

Where $\beta$ is FWHM and $\theta$ is Bragg's angle. For the AZO thin films the c-axis stress is calculated by employing the biaxial strain model(Jo et al., 2018):

$\sigma=\frac{2 c_{13}^{2}-c_{33}\left(c_{11}-c_{12}\right)}{2 c_{13}} \times \varepsilon$ 3
Where, $c_{11}, c_{12} c_{13}$ and $c_{13}$ are the elastic constants of a single crystal having the values 208.8, 114.7, 104.2 and 213.8 respectively. Equation (3) can be simplified to

$$
\sigma=-233 \times \varepsilon(G P a) .
$$

The micro-strain and stress value of the thin films are tabulated in Table1, the value first decreases for the $1 \% \mathrm{AZO}$ sample and then increase for the $2 \% \mathrm{AZO}$ sample. The positive sign of stress value refers to the tensile and negative sign for compressive stress(Jo et al., 2017). For undoped and 1\%AZO thin films, compressive stress is observed on the other hand tensile stress is obtained in the case of $2 \%$ AZO thin film. The augmented stress values, increase the light scattering in the $2 \% \mathrm{AZO}$ thin films and thus affecting the optical properties.

The FWHM of (002) peak, obtained from the XRD pattern are listed in Table2. The FWHM decrease for $1 \%$ AZO sample and then increases for $2 \% \mathrm{AZO}$ thin films as compared with undoped $\mathrm{ZnO}$ thin films. This result show improvement in the crystallinity for the $1 \% \mathrm{AZO}$ thin film. The average particle size $\mathrm{D}$ is calculated using Scherrer's formula(Srinatha et al., 2017) from the (002) peak.

Table 2: Optical bandgap value of pristine and implanted sample1\%AZO and 2\%AZO

\begin{tabular}{l|ccc} 
Al Conc. & Undoped ZnO & $\mathbf{1 \% A Z O}$ & $\mathbf{2 \% A Z O}$ \\
\hline Bandgap(eV) & 3.28 & 3.27 & 3.30 \\
& $D=\frac{K \lambda}{\beta \cos \theta}$
\end{tabular}


Where, $\mathrm{K}=0.9$ is Scherer's constant which depends on the shape of crystallite, $\lambda=1.5418 \AA$, is $\mathrm{X}$-ray wavelength for $\mathrm{Cu} \mathrm{K} \alpha$ target, $\beta$ is FWHM for (002) peak and $\theta$ is the Bragg's angle (half of the peak position angle). As the D value is calculated from the FWHM value it shows a similar trend with variation in Al dopant concentration. An increase in crystallite size for $1 \%$ AZO sample is a signature of reduction in $\mathrm{ZnO}$ stoichiometry by substitutional replacement of $\mathrm{Zn}$ with $\mathrm{Al}$

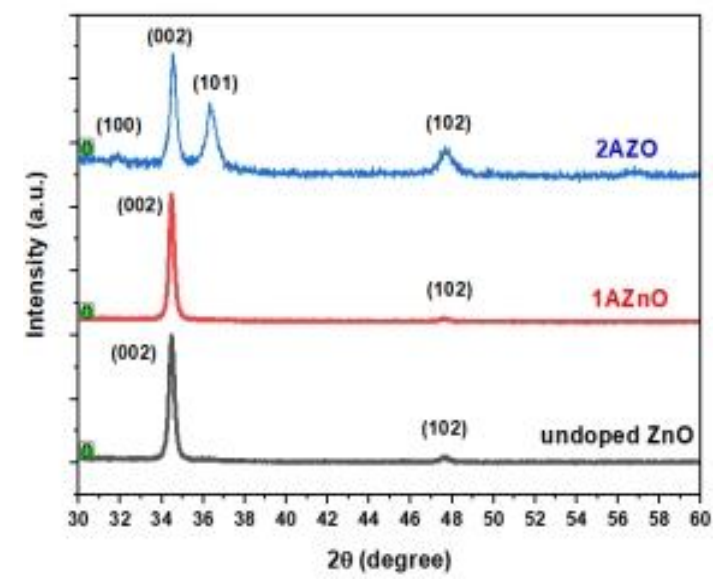

which is in harmony with the absence of any metallic phase. The variation in lattice parameter $\mathrm{c}$ with $\mathrm{Al}$ doping concentration from $1 \%$ to $2 \%$ alters the shape of the crystallite and can also be related to the variation in $\mathrm{D}$ value. This trend can be an outcome of the misfit stress that impose a negative effect on the structural factors of the thin films. The presence of the geometric mismatch between the deposited thin film and substrate is also responsible for these results.

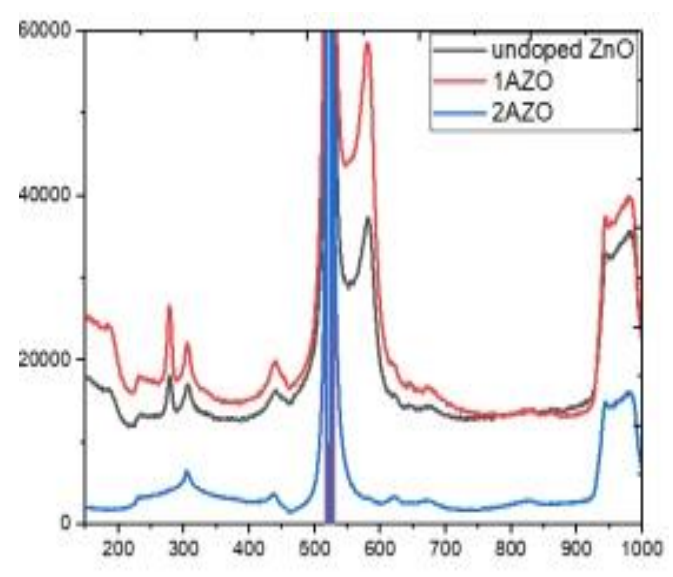

Figure 1: The XRD patterns of the undoped, $1 \% \mathrm{AZO}$ and $2 \% \mathrm{AZO}$ thin film samples Figure2: Micro-Raman spectra of undoped $\mathrm{ZnO}$ and $\mathrm{AZO}$ thin films

The length of dislocation lines per unit volume known as the dislocation density gives the extent of the defects in the crystal. The dislocation density $(\delta)$ has been estimated by employing the following formula(Yilmaz et al., 2016)

$$
\delta=\frac{1}{D^{2}}
$$

The values, given in Table1, decrease slightly for the $1 \%$ AZO sample and then increases for $2 \% \mathrm{AZO}$ thin films as compared with undoped $\mathrm{ZnO}$ thin films. The result indicates the increment of the defects in the crystal structure.

\subsection{Raman Studies}

Zinc oxide in wurtzite structure is in space group $\quad C_{V}^{6}$. The irreducible representation for the optical phonons in the Brillouin zone is given by Damen et al., 1966(Damen et al., 1966)

$$
\Gamma_{\text {optical }}=A_{1}+2 E_{2}+E_{1}
$$

where, $E_{1}$ and $A_{1}$ both are polar modes that split into longitudinal optical and transverse optical (TO), in the presence of macroscopic electric field related to (LO) phonons. The 
micro-Raman spectra of undoped $\mathrm{ZnO}$ and AZO thin films are shown in Figure2. The spectra consist of peaks at 440 and $580 \mathrm{~cm}^{-1}$, which are assigned to vibrational mode $\mathrm{E}_{2}$ (high) and $\mathrm{E}_{1}(\mathrm{LO})$ mode. The peak present at 306 and $522 \mathrm{~cm}^{-1}$ are due to Si substrate and emerged by two phonon modes and secondorder processes. The presence of this signal is due to lower film thickness than the penetration depth $(512 \mathrm{~nm})$ of the laser. These peaks are attributed to the combined (TO and TA) phonon modes 9 of Si. All the observed peaks of Raman spectra are within the error limit.

It is noteworthy that the profile and position of the Raman peak influenced by the presence of residual stress, crystallization, crystal defects and structural disorder in the thin film. The oxygen sublattice and wurtzite structure of $\mathrm{ZnO}$ is confirmed by $\mathrm{E}_{2}$ (high) mode(Gautam et al., 2014). The peak assigned for $E_{2}$ (high) mode persists in all the samples indicating the stable polycrystalline nature. The intensity is suppressed for a $2 \% \mathrm{AZO}$ sample showing the gradual increase of defect density (oxygen vacancies) with increasing $\mathrm{Al}$ concentration in $\mathrm{ZnO}$ thin films. The minute red shift in the frequency with a suppressed intensity of E2 mode can be correlated to the induced stress in the host lattice calculated by XRD results. The Raman peak emerging at $580 \mathrm{~cm}^{-1}$ is designated for $E_{1}(\mathrm{LO})$ mode in undoped $\mathrm{ZnO}$ and $1 \% \mathrm{AZO}$ thin films attributed to intrinsic defects $\left(\mathrm{V}_{\mathrm{O}}\right.$ and $\left.\mathrm{Zn}_{\mathrm{i}}\right)$ present in host $\mathrm{ZnO}$. This $\mathrm{E}_{1}(\mathrm{LO})$ mode is absent in $2 \%$ AZO thin film. The spectra indicate the presence of anomalous vibrational mode at $277 \mathrm{~cm}-1$ undoped $\mathrm{ZnO}$ and $1 \% \mathrm{AZO}$ sample whereas absent in $2 \%$ AZO sample. This mode is present in nitrogen-doped $\mathrm{ZnO}$. By theoretical calculation, Wang et al(Wang et al., 2006), suggest that this mode is due to vibrations of $\mathrm{Zn}$ adjacent to the $\mathrm{O}$ atoms that are replaced by the dopant $\mathrm{N}$ atom. However, the exact verification for the origin of this mode in our system still needs to be investigated.

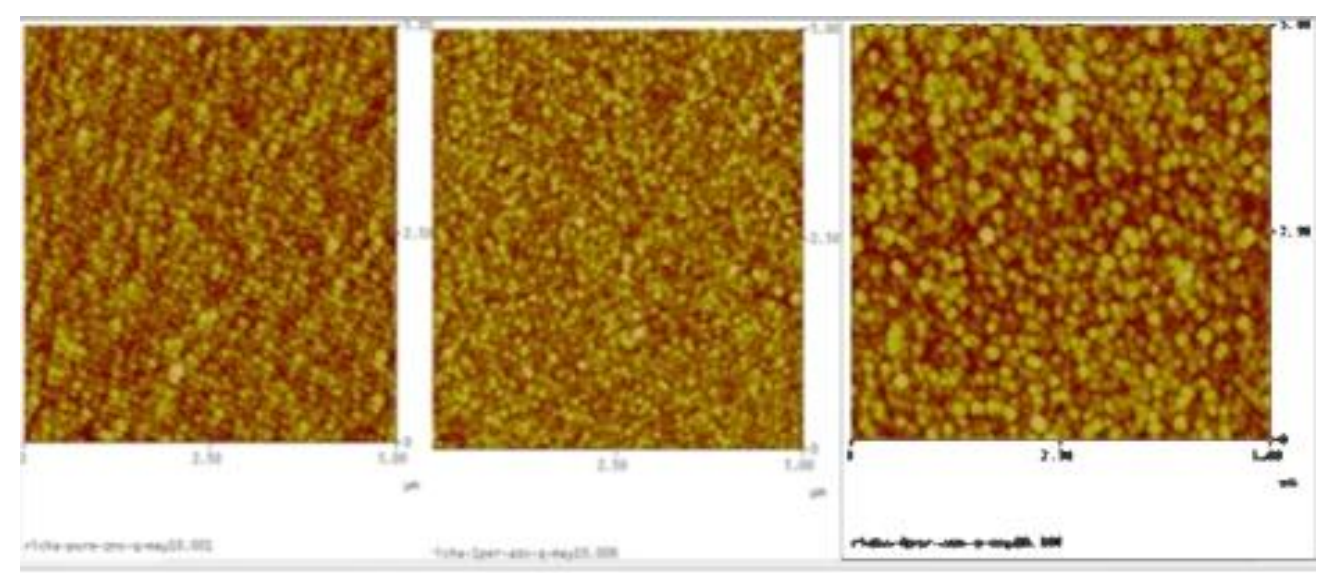

Figure 3: 2D AFM micrograph of (a) Undoped (b) 1\%AZO and (c) 2\%AZO thin film 


\section{Morphological Studies}

Atomic force microscopy is used to study the microstructure and morphology of the surface of the deposited thin films. The tapping mode AFM micrographs gives a comparison of undoped $\mathrm{ZnO}$ and $\mathrm{AZO}$ thin films are shown in Figure3.The dense grainy morphology with increased grain size for $2 \%$ AZO thin films. The topography of the undoped $\mathrm{ZnO}$ thin film is a relatively smooth and of good quality in contrast with the other thin films with increasing $\mathrm{Al}$ (1 and $2 \mathrm{wt} \%)$ amount. The surface roughness value shows a significant increase for $1 \% \mathrm{AZO}$ thin film $(8.811 \mathrm{~nm})$ and an increase in the case of $2 \%$ AZO $(7.404 \mathrm{~nm})$ thin film in comparison to undoped $\mathrm{ZnO}(6.158 \mathrm{~nm})$ thin films. The spherical shape grains in Undoped $\mathrm{ZnO}$ thin films get distorted which is attributed to increasing $\mathrm{Al}$ (1 and 2 wt \%) amount for $1 \% \mathrm{AZO}$ and $2 \% \mathrm{AZO}$ thin films. These results are also consistent with the XRD results. The surface morphologies show a clear and noticeable change with $\mathrm{Al}$ doping. Figure 3 shows that the undoped $\mathrm{ZnO}$ thin film entails consistent and symmetrical spherical crystallites of uniform size in contrast to distorted spheres in the case of AZO thin films.

This change in the crystallite shape may be attributed to the transition of compressive stress to tensile stress in the case of undoped $\mathrm{ZnO}$ and $2 \% \mathrm{AZO}$ thin films respectively. With an upsurge in $\mathrm{Al}$ concentration from $1 \mathrm{wt} \%$ to $2 \mathrm{wt} \%$, the grains grow into more of a random shape. The films become more densely packed without any cracks on the surface. Larger grains are observed for $2 \%$ AZO thin film. The particle size has been calculated from FE-SEM micrographs (Figure 4). The average particle size is about 46, 67 and $83 \mathrm{~nm}$ for undoped, $1 \% \mathrm{AZO}$ and $2 \% \mathrm{AZO}$ thin films respectively. The trend of increase in particle size is in harmony with the XRD results but as expected XRD analysis underestimated the crystallite size. Thus we can say that the concentration of dopant affects the morphology of the thin films.

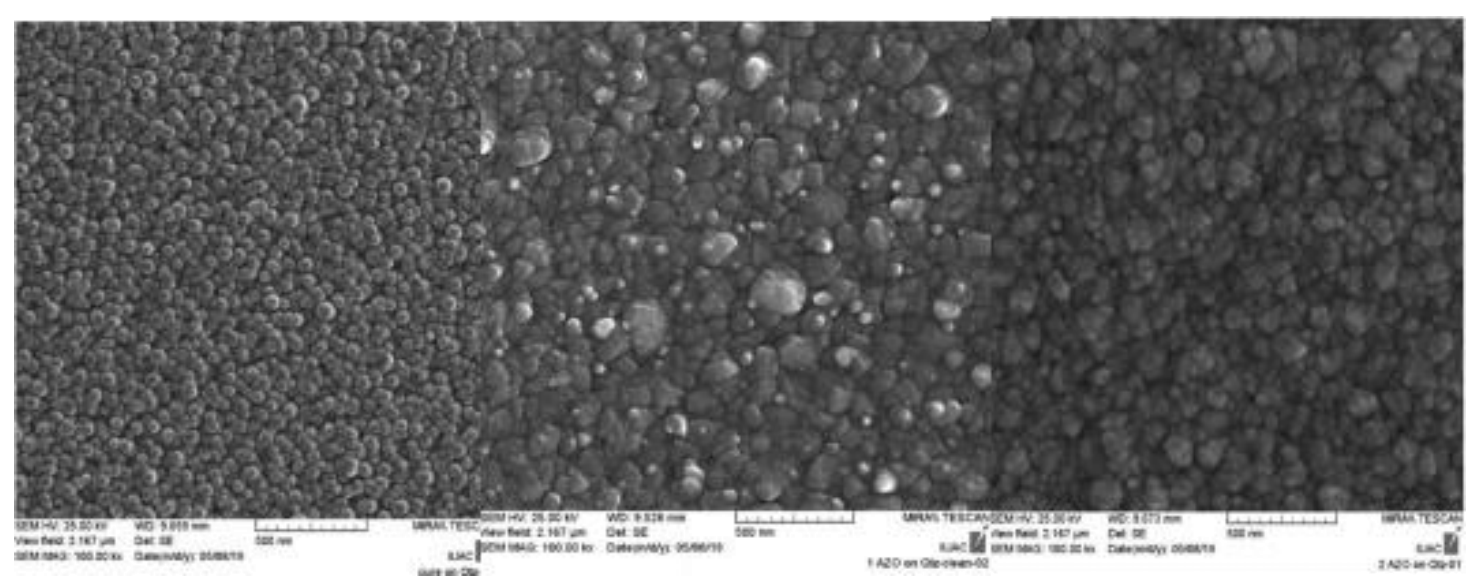

Figure4: FE- SEM micrograph of (a) Undoped (b) 1\%AZO and (c) 2\%AZO thin film 


\section{Optical Studies}

In order to study the effects of $\mathrm{Al}$ dopant concentration on the optical properties of the AZO thin films, the UV-Vis spectrophotometer is employed to record the optical transmittance and absorbance spectra. The Transmittance spectra are depicted in Figure 5. Particularly all the deposited thin films are transparent in the visible region with average transmittance lies between $80-90 \%$. The transmittance pattern shows an interference pattern originating due to the interference of light at the boundary of the substrate and deposited films which verifies the smoothness of the deposited thin films. The transmittance of the $1 \%$ AZO sample decrease significantly which further increase for $2 \% \mathrm{AZO}$ thin films. This change in the transmittance can be attributed to the variation in $\mathrm{Al}$ dopant concentration and particle size, witnessed by the other characterization method.

In semiconductors the optical transitions information (optical band gap) can be extracted from the basic absorbance spectra by the optical method and illustrated in figure6. The optical bandgap is calculated using Tauc's equation(Tauc et al., 1966)

$$
\alpha h v \approx C\left(h v-E_{g}\right)^{1 / 2} \quad 7
$$

where $\mathrm{C}$ is a constant, $\alpha$ is the absorption coefficient, $v$ is the photon energy and $1 / 2$ is for the direct transitions. The values are tabulated in Table 2. With reference to deposited undoped $\mathrm{ZnO}$ thin films the bandgap of $1 \%$ AZO thin-film decrease slightly and increase with increased $\mathrm{Al}$ concentration for $2 \%$ AZO thin film. The obtained values of optical bandgap are in harmony with the values in the literature despite the various thin film deposition method. The increase in the bandgap value with increased $\mathrm{Al}$ concentration can be understood by increased carrier concentration. In the substitution of host $\mathrm{Zn}^{2+}$ ions by $\mathrm{Al}^{3+}$ dopant ion, an extra free electron is available which occupy the energy levels that lies below the conduction band. It is a well-known fact that an augmentation in the optical band gap of the metal oxide semiconductor is observed with increasing carrier concentration and is well explained by the BM effect.

In contrast, there are some other factors such as the crystallite size, phase purity internal stress etc. which influence the optical band gap and may responsible for the observed decrement in the case of $1 \% \mathrm{AZO}$ thin film(Zhu et al., 2011). It can be concluded by the observed results that the bandgap can be controlled by altering the $\mathrm{Al}$ concentration.

\section{Electrical Transport Studies}

In semiconductors, mobility is an imperative factor as it explains the movement of the particle in the electric field. The n-types conductivity, in $\mathrm{ZnO}$, arises due to the oxygen vacancies and Zinc interstitials. The Undoped $\mathrm{ZnO}$ thin films prepared by $\mathrm{RF}$ sputtering method are highly resistive and does not show ohmic behaviour in the I-V curve.

On the other hand, the $\mathrm{AZO}(1 \%$ and $2 \%$ ) thin films are n-type conductive with ohmic nature measured by using Keithley two 
probe set up and are shown in Figure7. The mean resistivity value of $1 \% \mathrm{AZO}$ and $2 \% \mathrm{AZO}$ thin-film is $1.48 \times 10^{8} \mathrm{ohm}$ and $2.58 \times 10^{9} \mathrm{ohm}$ respectively. The effective substitution of $\mathrm{Al}$ dopant ion reduces the strained bonds and dangling between the Zinc ion and oxygen vacancies, which in turns reduce the resistivity value of $1 \% \mathrm{AZO}$ thin film as compared to undoped $\mathrm{ZnO}$. This substitution also increases the carrier concentration in the thin films resulting in an improvement in the conductivity.

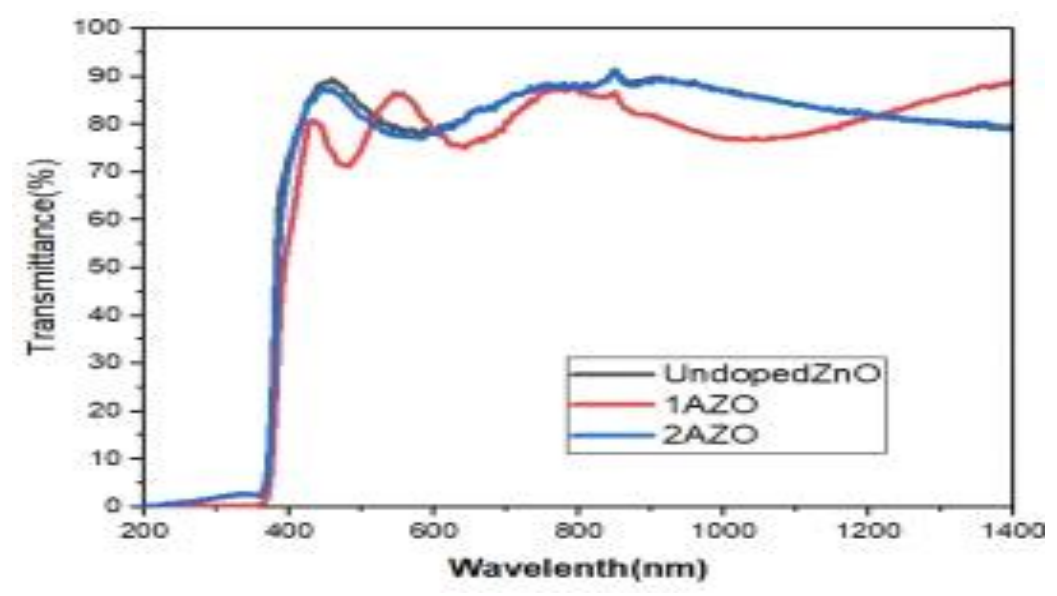

Figure 5: Transmission curve of undoped and AZO thin films

The $\mathrm{Al}$ dopant (donor $\mathrm{Al}^{3+}$ cation) shifts the Fermi level of AZO thin films to the higher position into the conduction band due to which completely degenerate films are obtained. The preferred c-axis (002) orientation as observed by the XRD pattern of the thin films also collaborates with the resistivity values. The $1 \%$ AZO thin films have lower resistivity value owning to the crystalline nature and more preferable c- axis (002) orientation as compared to the $2 \%$ AZO thin films. These results are well reported in literature(Srinatha et al., 2017). The lower resistivity value for $1 \%$ AZO thin films can be understood by reduced lattice defect that is also confirmed by XRD results and increased scattering of carrier electron from the grain boundaries. The free charge electrons can be trapped by the grain boundaries and a potential barrier is formed, which scatters the conduction electrons. This scattering results in the accumulation of negative charge which increases the resistivity value in $2 \% \mathrm{AZO}$ thin films. Apart from the crystal orientation the crystallite size also affects the resistivity of the thin film. This trend shown by the resistivity values can easily be correlated with the crystallite size calculated by XRD data. As the crystallite size increases it reduces the grain boundaries causing the decrement in the resistivity. The change in grain size can also be observed from the SEM images and shows the same trend. 


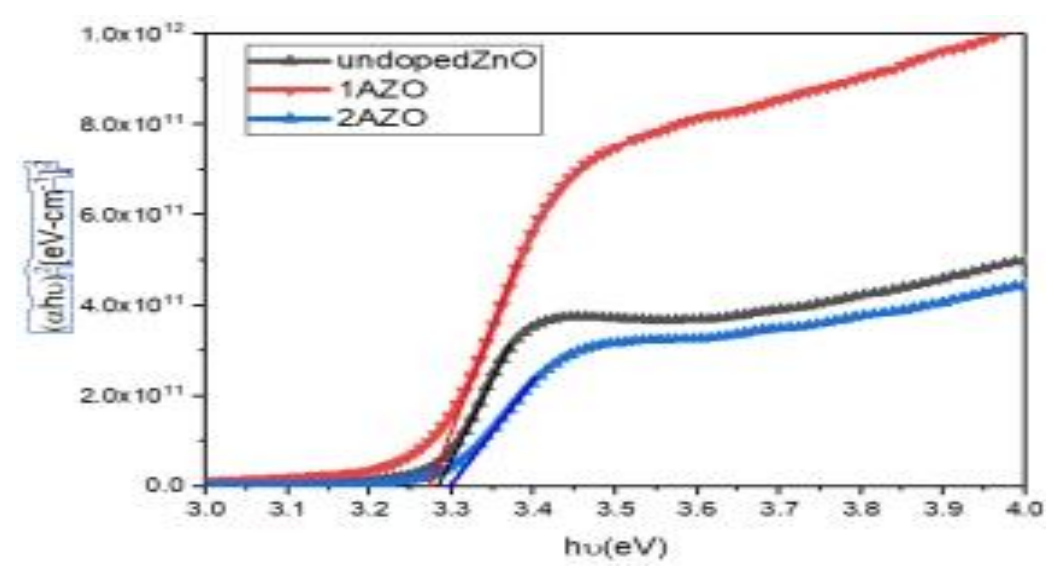

Figure 6: Tauc plot of undoped and AZO thin film

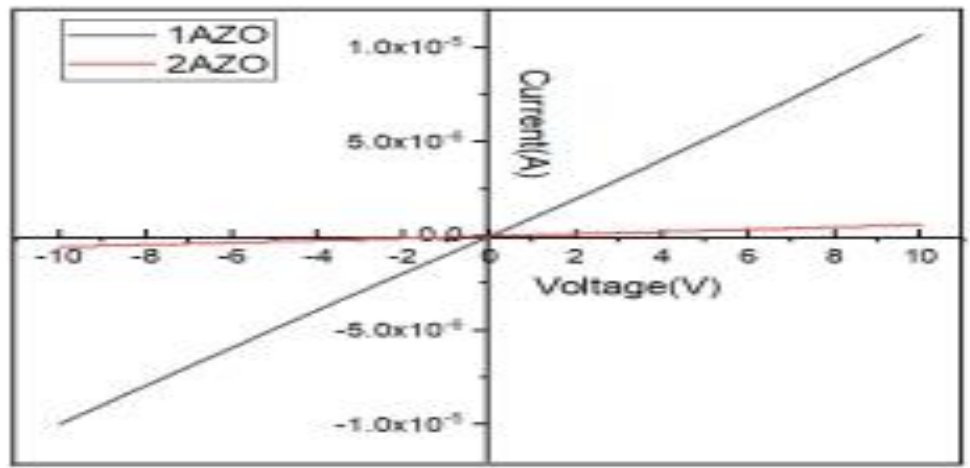

Figure $7: \mathrm{I}-\mathrm{V}$ plot of $1 \% \mathrm{AZO}$ and $2 \% \mathrm{AZO}$ thin film sample

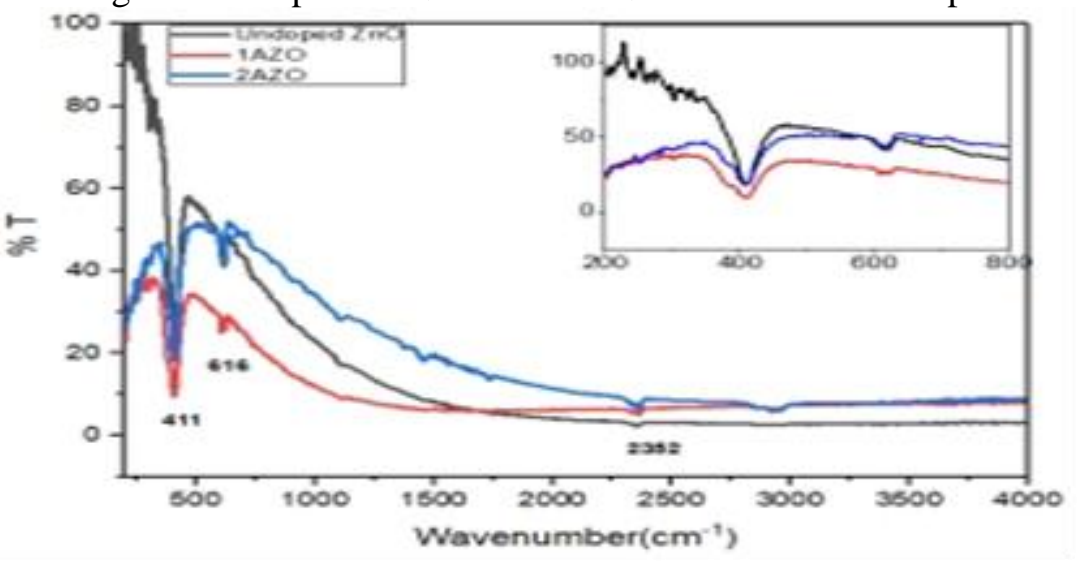

Figure 8: FTIR spectrum of Undoped $\mathrm{ZnO}, 1 \% \mathrm{AZO}$ and 2\%AZO thin films

\section{FTIR Studies}

Fourier-transform infrared spectroscopy (FTIR) is a useful technique to characterise the functional groups, vibrational properties, defect and impurities of the synthesized materials. The structure, morphology and chemical composition of the thin films decides the position of the absorption peak and band position. Figure8 shows the FTIR spectrum of Undoped $\mathrm{ZnO}, 1 \% \mathrm{AZO}$ and $2 \% \mathrm{AZO}$ thin films recorded in the range. In the IR spectrum, a signature peak is observed at 411 
$\mathrm{cm}^{-1}$ for the undoped $\mathrm{ZnO}$ and $\mathrm{AZO}$ thin films and corresponds to the $\mathrm{ZnO}$ bond stretching in tetrahedral coordination.

Broadening in the peak is observed due to the doping effect which may be attributed to the stress caused by the Al ion. The stretching vibrations of $\mathrm{Zn}-\mathrm{O}$ bonds present in octahedral coordination originate the peaks around $617 \mathrm{~cm}^{-1}$. Although these peaks are present in the spectrum but are not as prominent as peaks originated by the tetrahedral coordination of $\mathrm{ZnO}$ which further confirms the wurtzite structure of the deposited thin film sample. This FTIR observation is in harmony with the XRD analysis of the samples. The position and broadness of the signal at $617 \mathrm{~cm}^{-1}$ is not much affected by the doping concentration, therefore it confirms that the substitution of $\mathrm{Zn}$ ion by the $\mathrm{Al}$ ions in the tetrahedral arrangement. $\mathrm{A}$ weak peak at $1102 \mathrm{~cm}^{-1}$ is due to the substrate Si-O-Si bonds. In a $2 \%$ AZO thin film sample, a weak peak is observed at $1458 \mathrm{~cm}^{-1}$ assigned to the symmetrical $\mathrm{C}=\mathrm{O}$ stretching of zinc carboxylate due to Lewis acidity(Sharma and Jha, 2017). The atmospheric $\mathrm{CO}_{2}$ absorption gives rise to the peak at $2373 \mathrm{~cm}^{-1}$. A broadband at $2922 \mathrm{~cm}^{-1}$ can be observed in $2 \%$ AZO thin-film originating due to the presence of $\mathrm{CH}$. The presence of these band can be related to the absorbed group on the thin film surface and does not reflect the contaminations.

\section{Conclusion}

The undoped $\mathrm{ZnO}, 1 \% \mathrm{AZO}$ and $2 \% \mathrm{AZO}$ thin films are deposited on quartz substrate by using RF magnetron sputtering. XRD results confirm the presence of highly textured and polycrystalline $\mathrm{ZnO}$ thin films with preferential c-axis orientation. The result indicates that as we increase $\mathrm{Al}$ concentration in $\mathrm{ZnO}$ the nature of stress is changed, which in turn affect the optical properties. The crystallinity of the thin film is enhanced but at the same time, the number of defects is also increased in the thin film. Raman study indicates that the profile and position of the Raman peak are influenced by the alteration in residual stress, crystallization, crystal defects and structural disorder that is present in the thin film. The percentage of $\mathrm{Al}$ content clearly affects the surface roughness that is depicted in AFM analysis. The increase in particle size calculated from FE-SEM is consistent with the crystallite value calculated in XRD analysis. The average transmittance of all the thin films is $80-90 \%$ in the visible region. The increase in optical bandgap under different doping percentage is explained by the $\mathrm{BM}$ effect. The ohmic behaviour of doped thin film is observed by two probe setup. The resistivity value is altered with $\mathrm{Al}$ doping. The presence of different functional groups, defects and impurities is confirmed by FTIR. The results clearly show that the introduction of $\mathrm{Al}$ dopant alters the various $\mathrm{ZnO}$ material properties.

\section{Acknowledgements}

The authors are grateful to the Director, Inter University Accelerator Centre, New Delhi for providing support throughout the experimental work. The authors are also thankful to $\mathrm{Dr}$ 
Pawan Kularia, Dr S.A. Khan and Dr Indira Sulani for experimental support. One of the authors (RS) is also thankful to UGC for providing the financial support under the scheme of $\mathrm{PhD}$ student fellowship.

\section{References}

Damen, T.C., Porto, S.P.S., Tell, B., 1966. Raman effect in zinc oxide. Phys. Rev. 142, 570.

Gautam, S.K., Singh, F., Sulania, I., Singh, R.G., Kulriya, P.K., Pippel, E., 2014. Micro-Raman study on the softening and stiffening of phonons in rutile titanium dioxide film: Competing effects of structural defects, crystallite size, and lattice strain. J. Appl. Phys. 115, 143504.

Granqvist, C.G., 2000. Electrochromic tungsten oxide films: review of progress 1993-1998. Sol. Energy Mater. Sol. Cells 60, 201-262.

Jo, G.H., Kim, S., Koh, J., 2017. Enhanced electrical and optical properties based on stress reduced graded structure of Al-doped $\mathrm{ZnO}$ thin fi lms Al doping $\mathrm{d}$ co tion ( mol \%. Ceram. Int. 0-1. https://doi.org/10.1016/j.ceramint.2017.0 9.240

Jo, G.H., Kim, S.H., Koh, J.H., 2018. Enhanced electrical and optical properties based on stress reduced graded structure of Al-doped $\mathrm{ZnO}$ thin films. Ceram. Int. 44, 735-741. https://doi.org/10.1016/j.ceramint.201 7.09 .240
Kaur, G., Mitra, A., Yadav, K.L., 2015. Influence of Beam Energy on the Properties of Pulsed Laser Deposited AlDoped $\mathrm{ZnO}$ Thin Films. IEEE Trans. Nanotechnol. $\quad 14, \quad 922-930$. https://doi.org/10.1109/TNANO.2015.246 3085

Kim, K.H., Park, K.C., Ma, D.Y., 1997. Structural, electrical and optical properties of aluminum doped zinc oxide films prepared by radio frequency magnetron sputtering. J. Appl. Phys. 81, 7764-7772.

https://doi.org/10.1063/1.365556

Lee, J.H., Park, B.O., 2004. Characteristics of Al-doped $\mathrm{ZnO}$ thin films obtained by ultrasonic spray pyrolysis: Effects of $\mathrm{Al}$ doping and an annealing treatment. Mater. Sci. Eng. B Solid-State Mater. Adv. Technol. 106, 242-245. https://doi.org/10.1016/j.mseb.2003.09.04 0

Malek, M.F., Mamat, M.H., Sahdan, M.Z., Zahidi, M.M., Khusaimi, Z., Mahmood, M.R., 2013. Influence of various sol concentrations on stress/strain and properties of $\mathrm{ZnO}$ thin films synthesised by sol-gel technique. Thin Solid Films 527, 102-109.

Peiró, A.M., Ravirajan, P., Govender, K., Boyle, D.S., O'Brien, P., Bradley, D.D.C., Nelson, J., Durrant, J.R., 2006. Hybrid polymer/metal oxide solar cells based on $\mathrm{ZnO}$ columnar structures. J. Mater. Chem. 16, 2088-2096.

Sharma, D., Jha, R., 2017. Analysis of 
structural, optical and magnetic properties of $\mathrm{Fe} / \mathrm{Co}$ co-doped $\mathrm{ZnO}$ nanocrystals. Ceram. Int. 43, 8488-8496. https://doi.org/10.1016/j.ceramint.2017.0 3.201

Srinatha, N., Raghu, P., Mahesh, H.M., Angadi, B., 2017. Spin-coated Al-doped $\mathrm{ZnO}$ thin films for optical applications: Structural, micro-structural, optical and luminescence studies. J. Alloys Compd. 722, 888-895. https://doi.org/10.1016/j.jallcom.2017.06. 182

Tauc, J., Grigorovici, R., Vancu, A., 1966. Optical properties and electronic structure of amorphous germanium. Phys. status solidi 15, 627-637.

Van der Drift, A., 1967. Evolutionary selection, a principle governing growth orientation in vapour- deposited layers. Philips Res. Rep 22, 267.

Wang, J.B., Zhong, H.M., Li, Z.F., Lu, W., 2006. Raman study of N+-implanted ZnO. Appl. Phys. Lett. 88, 101913.

Yilmaz, M., Tatar, D., Sonmez, E., Cirak, C., Aydogan, S., Gunturkun, R., 2016. Investigation of Structural, Morphological, Optical, and Electrical Properties of Al Doped ZnO Thin Films Via Spin Coating Technique. Synth. React. Inorganic, Met. Nano-Metal Chem. 46 , 489-494. https://doi.org/10.1080/15533174.2014.98 8795

Zhu, B.L., Wang, J., Zhu, S.J., Wu, J., Wu, R., Zeng, D.W., Xie, C.S., 2011. Influence of hydrogen introduction on structure and properties of $\mathrm{ZnO}$ thin films during sputtering and post- annealing. Thin Solid Films 519, 3809-3815. 